1Secretaria de Saúde Ipojuca (PE), Brasil. gilcele_@hotmail.com

2 Instituto de Medicina Integral Professor Fernando Figueira (Imip) - Recife (PE), Brasil.

\section{Avaliação da implantação de um Centro de Atenção Psicossocial em Pernambuco, Brasil}

\author{
Evaluation of the implementation of a Psychosocial Care Center in \\ Pernambuco, Brazil
}

Gilcele Marília da Silva', Eronildo Felisberto², Isabella Samico ${ }^{2}$, Ana Coelho de Albuquerque ${ }^{\mathbf{2}}$

DOI: $10.1590 / 0103-1104202012714$

RESUMO O estudo teve como objetivo avaliar a implantação do Centro de Atenção Psicossocial II (Caps II) de um município do estado de Pernambuco. Trata-se de uma pesquisa avaliativa, de análise de implantação, relacionando a variação do grau de implantação do Caps sobre os efeitos observados. Elaborou-se modelo lógico da intervenção e matriz de indicadores e julgamento. Realizaram-se entrevistas individuais, observação direta e análise de registros e documentos. Consideraram-se os indicadores de estrutura e processo para análise do grau de implantação, posteriormente relacionado aos indicadores de resultados. O serviço foi classificado como parcialmente implantado (74,4\%), com grau de implantação de 68,0\% na dimensão estrutura e de $80,9 \%$ na dimensão processo, classificadas como parcialmente implantado e implantado, respectivamente. Os componentes assistência à saúde e mobilização e educação na saúde foram considerados parcialmente implantados, ao passo que o componente gestão foi considerado implantado. Os indicadores de resultado demonstraram coerência com o grau de implantação, especialmente em relação ao processo. Mesmo que a estrutura do Caps tenha apresentado algumas deficiências, não exerceu influência direta nos resultados. Concluiu-se que o Caps é um equipamento importante para rede local, embora necessite superar desafios estruturais a fim de contribuir com a articulação estratégica da política de saúde mental do município.

PALAVRAS-CHAVE Saúde mental. Serviços de saúde mental. Avaliação em saúde. Avaliação de serviços de saúde.

ABSTRACT The study aimed to evaluate the implementation of the Psychosocial Care Center II (Caps II) in a municipality in the state of Pernambuco. It is an evaluative research, of implementation analysis, relating the variation of the degree of implementation of the Caps on the observed effects. Logic model of intervention and matrix of indicators and judgment were elaborated. Individual interviews, direct observation and analysis of records and documents were carried out. Structure and process indicators were considered to analyze the degree of implementation, which is related to the result indicators. The service was classified as partially implemented (74.4\%), with a $68.0 \%$ degree of implementation in the structure dimension and $80.9 \%$ in the process dimension, classified as partially implemented and implemented, respectively. The health care and mobilization and health education components were considered partially implemented, while the management component was considered implemented. The result indicators showed consistency with the degree of implementation, especially in relation to the process. Even though the Caps structure has some deficiencies, they did not have a direct influence on the achievement of results. It was concluded that the Caps is an important equipment for the local network, but it needs to overcome structural challenges in order to contribute to the strategic articulation of the municipality's mental health policy.

KEYWORDS Mental health. Mental health services. Health evaluation. Evaluation of health services. 


\section{Introdução}

Os transtornos mentais e comportamentais atingem cerca de 700 milhões de pessoas no mundo, submetendo-as ao risco de violação de direitos humanos, isolamento e exclusão social, baixa qualidade de vida, além de elevados custos dentro e fora do sistema de saúde'. No Brasil, os dados revelam que $3,0 \%$ da população geral sofre com transtornos mentais graves e persistentes; $6,0 \%$ apresenta transtornos psiquiátricos graves decorrentes do uso de álcool e outras drogas; e $12,0 \%$ necessita de algum atendimento, seja ele contínuo ou eventual².

A trajetória da assistência psiquiátrica está vinculada à internação de usuários em manicômios, num processo de exclusão e alienação. No entanto, desde o final da década de 1960, essa realidade vem passando por um movimento de reestruturação dos saberes e de suas práticas, que culminaram, na década de 1970, na reforma psiquiátri$\mathrm{ca}^{3}$. Posteriormente, no final dos anos de 1980, o movimento da reforma psiquiátrica desenvolveu o pensamento crítico em relação à institucionalização da loucura e, em 1986, foi criado o primeiro Centro de Atenção Psicossocial (Caps) do Brasil. Porém, somente com a Lei Paulo Delgado ${ }^{\circ}$ 10.216, de 06 de abril de 2001, oficializou-se o direito das pessoas portadoras de transtornos mentais, com o redirecionamento do modelo assistencial em saúde mental no país ${ }^{4,5}$.

Assim, a Política Nacional de Saúde Mental vigente visa a reduzir progressivamente os leitos em hospitais psiquiátricos, expandindo, qualificando e fortalecendo a rede extra-hospitalar mediante a implantação dos serviços substitutivos da Rede de Atenção Psicossocial (Raps). Tais serviços funcionam de portas abertas e estão localizados onde as pessoas vivem, nos seus bairros, onde moram suas famílias, seus amigos e próximos aos locais frequentados pelas pessoas ${ }^{6}$.
Nesse contexto, estão os Caps, espaços de construção contínua de um novo fazer, no qual se privilegia o sujeito com sofrimento e não a doença em si. A equipe multiprofissional dos Caps deve direcionar suas intervenções no sentido de reabilitar o indivíduo, reintegrando-o à sua família e à comunidade. Além disso, tem por atribuições a responsabilização pela organização da demanda e da rede de cuidados em saúde mental no âmbito de seu território, supervisão das equipes de atenção básica, entre outras ${ }^{7}$.

No entanto, mesmo após a Reforma Psiquiátrica, velhos e novos obstáculos emergem, a exemplo da reprodução de práticas manicomiais nos serviços substitutivos. No plano micropolítico, observam-se dificuldades quanto à ruptura de práticas profissionais que afirmam a objetificação do portador de sofrimento psíquico, a supressão do sintoma, os 'especialismos', a demanda por internação, a não continuidade do cuidado, a 'ambulatorização' da assistência, ou seja, processos de trabalho que acabam mantendo a cultura manicomial em vigor ${ }^{8}$.

Portanto, a proposta de estudos avaliativos torna-se pertinente e atual frente aos obstáculos apresentados. Bessa e colaboradores7, avaliando a assistência de saúde mental em um Caps do interior do Brasil, observaram que há fragilidades importantes na condução terapêutica do serviço em três domínios pesquisados: processo ou atividades terapêuticas; processo ou gestão do cuidado; e atividades comunitárias ou externas ao Caps. A variação de carga horária dos profissionais parece impactar negativamente a assistência, além de fatores como o modelo de atenção centrado na doença, que dificulta a autonomia dos usuários ${ }^{9}$.

Além disso, avaliar os serviços de saúde mental pode contribuir para que os profissionais de saúde, juntamente com os órgãos competentes, desenvolvam ações especializadas de atendimento aos usuários, a fim 
de proporcionar condições mais humanizadas, visando à qualidade da assistência. Considerando que a análise das práticas de saúde constitui ferramenta importante para a transformação da realidade, tendo em vista e as diretrizes que orientam o modelo de atenção em saúde mental no campo psicossocial, este estudo teve como objetivo avaliar a implantação do Caps II de um município do estado de Pernambuco.

\section{Material e métodos}

Trata-se de uma pesquisa avaliativa do tipo análise de implantação do componente 2 , que relaciona a influência da variação do Grau de Implantação (GI) do Caps sobre os efeitos observados ${ }^{10}$. Com isso, objetivou-se fazer um julgamento sobre a intervenção, comparando os recursos empregados com a sua organização, ou estrutura, com os serviços ou bens produzidos, ou seu processo, e com os resultados obtidos. A estratégia de investigação adotada foi o estudo de caso único ${ }^{11}$ e o Caps II foi a intervenção.

A pesquisa foi realizada no Caps II de Bezerros, município localizado na mesorregião do agreste de Pernambuco, e os dados foram coletados durante o período de janeiro a maio de 2019. Segundo a Portaria $n^{\circ} 336$, de 19 de fevereiro de 2002, o Caps II possui capacidade operacional para atendimento a municípios com população entre 70 mil e 200 mil habitantes, devendo funcionar os dois turnos durante cinco dias na semana. A assistência prestada ao paciente no Caps II inclui atendimento individual, atendimento a grupos, atendimento em oficinas terapêuticas, visitas domiciliares, atendimento à família e atividades comunitárias com foco na integração do doente mental na comunidade e sua inserção familiar e social. Tais atividades são coerentes com o paradigma no qual se fundamenta a Reforma Psiquiátrica ${ }^{12}$.
O Caps II de Bezerros foi implantado no mês de fevereiro de 2015 e, no momento da pesquisa, possuía 110 usuários admitidos e funcionando de acordo com o preconizado. As marcas históricas da assistência em saúde mental de Bezerros acompanham a tendência hegemônica dos municípios de pequeno porte do Brasil. Foi com a implantação do Caps II que se iniciou o processo de articulação estratégica da Política Municipal de Saúde Mental, sendo um dispositivo importante da Raps.

Os usuários admitidos no serviço são, preferencialmente, pessoas com transtornos mentais severos ou persistentes, dos tipos transtornos graves do comportamento, primeira crise, constantes reinternações, psicóticos senis sem sintomatologia demencial, neuróticos graves, oligofrênicos leves com instabilidade na conduta social e ou sintomatologia psiquiátrica aguda, usuários com transtorno mental devido ao uso abusivo de álcool e outras drogas. Os pacientes são identificados durante o acolhimento que acontece diariamente no serviço, momento em que é estabelecido o primeiro contato com a equipe. A prática terapêutica do serviço consta de acolhimento e assistência ao usuário e seus familiares, partindo de uma atenção interdisciplinar onde a escuta e a compreensão do sofrimento psíquico devem orientar a elaboração do Projeto Terapêutico Singular (PTS).

Para explicitar a intervenção avaliada, o Caps II, delineou-se o modelo lógico a partir da tríade estrutura-processo-resultado Donabedian ${ }^{13}$, com base em três componentes: assistência à saúde, gestão do Caps, e mobilização social e educação na saúde. A modelização expõe a forma como os componentes se articulam por meio de atividades desenvolvidas e recursos disponíveis para alcançar os resultados $^{10}$. Para descrever a organização do Caps II do município de Bezerros, uma primeira versão do modelo lógico foi elaborada tomando como referência os documentos e legislações dispostos no quadro 1. 
Quadro 1. Documentos técnicos utilizados para a construção do modelo lógico do Centro de Atenção Psicossocial - Caps II. Município de Bezerros - Pernambuco, 2019

\section{Documento}

BRASIL, 2013 - Secretaria de Atenção à Saúde

BRASIL, 2011 - Centro de Estudo e Pesquisa em Saúde Coletiva

BRASIL, 2014 - Universidade Federal de Santa Catarina

Portaria no 3.588/GM/MS de 21 de dezembro de 2017

Fonte: Elaboração própria.

Caps - Centro de Atenção Psicossocial; UA - Unidade de Acolhimento.

\section{Dispõe sobre}

Manual de estrutura física dos Centros de Atenção Psicossocial e Unidades de Acolhimento: orientações para elaboração de projetos de construção de Caps e de UA como lugares da atenção psicossocial nos territórios

Guia prático de matriciamento em saúde mental

Crise e urgência em saúde mental: organização da atenção psicossocial à crise em rede de cuidado

Dispõe sobre a Rede de Atenção Psicossocial
O modelo inicialmente proposto foi submetido à análise por especialistas em saúde mental ( $n=6)$ e avaliação em saúde $(n=2)$, que receberam por meio eletrônico a versão preliminar e puderam acrescentar ou suprimir informações que consideraram necessárias. O processo aconteceu em duas rodadas e o modelo foi considerado finalizado quando os especialistas não tiveram mais alterações a fazer (figura 1). 
Figura 1. Modelo lógico do Centro de Atenção Psicossocial - Caps II. Município de Bezerros - Pernambuco, 2019

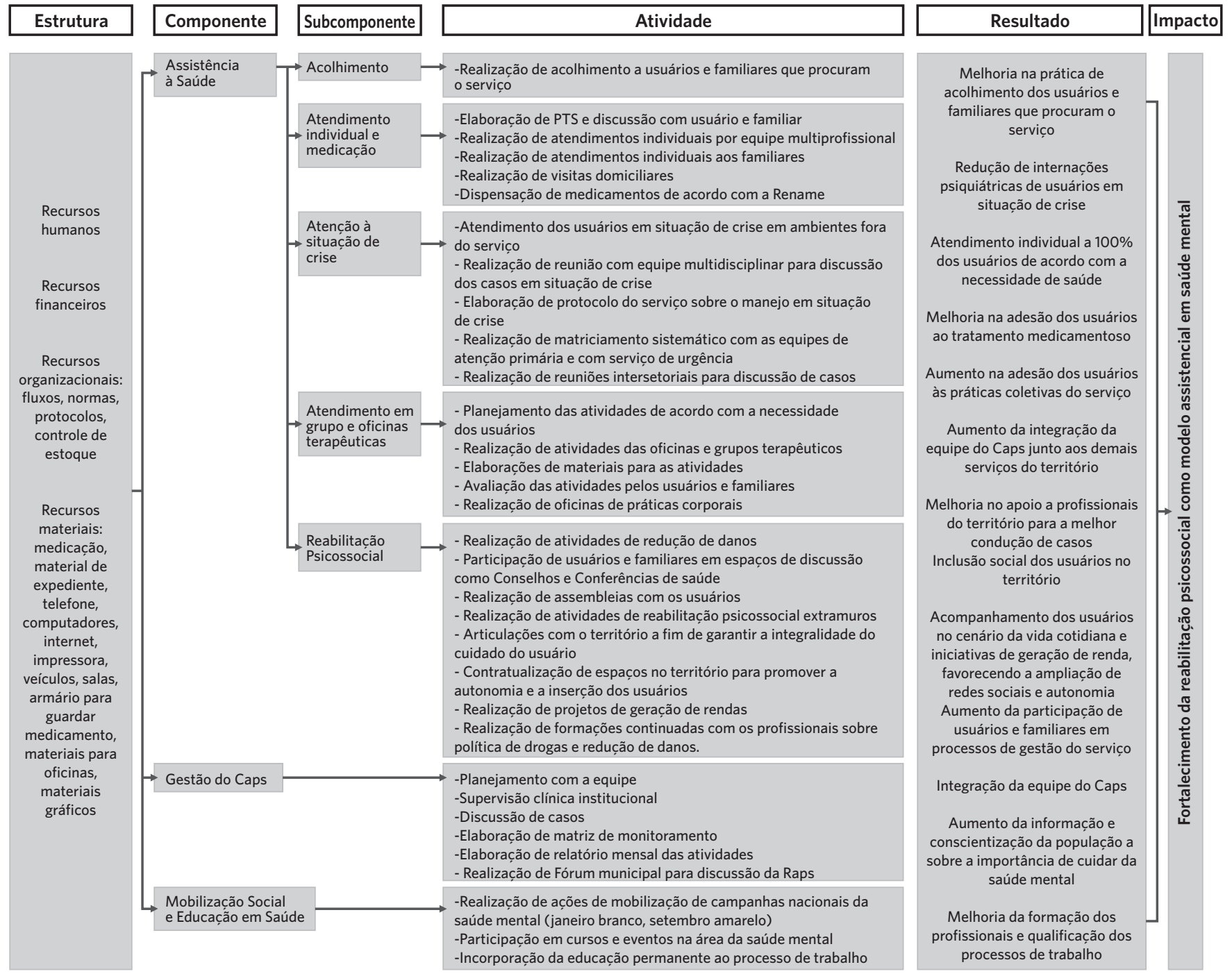

Em seguida, foi elaborada a matriz de indicadores e julgamento com base no modelo lógico, que também foi submetida à análise pelos especialistas supracitados. Na matriz, encontram-se, por componente e subcomponente, a pontuação e a fonte de cada indicador (quadro 2). 
Quadro 2. Matriz de indicadores e julgamento, segundo componentes, subcomponentes e dimensões de estrutura e processo. Centro de Atenção Psicossocial - Caps II. Município de Bezerros - Pernambuco, 2019

\begin{tabular}{|c|c|c|c|}
\hline Dimensão & Indicador & Pontuação & Parâmetros \\
\hline \multicolumn{4}{|c|}{ Componente: Assistência à Saúde } \\
\hline \multicolumn{4}{|c|}{ Subcomponente: Acolhimento } \\
\hline \multirow[t]{9}{*}{ Estrutura } & Existência de protocolo com diretrizes do Caps & $\begin{array}{l}\text { Sim e disponível = 1,5 / Sim, mas não está disponível = } \\
1,0 / \text { Não }=0,0\end{array}$ & SIM \\
\hline & $\begin{array}{l}\text { Sala para realização de acolhimento com birô e ca- } \\
\text { deiras }\end{array}$ & $\operatorname{Sim}=1,0 /$ Não $=0,0$ & SIM \\
\hline & $\begin{array}{l}\text { Horário de funcionamento do serviço adequado e de } \\
\text { acordo com a legislação }\end{array}$ & $\begin{array}{l}\text { Sim, funcionamento de 8h/dia, sem fechar no horário } \\
\text { de almoço= } 1,0 / \mathrm{Sim} \text {, funcionando } 8 \mathrm{~h} / \text { dia, mas fechan- } \\
\text { do no horário de almoço= 0,5/ Não funciona 8h/dia = } \\
0,0\end{array}$ & $\begin{array}{l}\text { SIM, funcionan- } \\
\text { do } 8 \text { h/dia, sem } \\
\text { fechar para o } \\
\text { almoço }\end{array}$ \\
\hline & $\begin{array}{l}\text { Existência de listagem das ações e atividades oferta- } \\
\text { das pelo serviço }\end{array}$ & Sim e disponível $=0,5 /$ Não $=0,0$ & SIM e disponível \\
\hline & Televisão funcionando & $\operatorname{Sim}=0,5 /$ Não $=0$ & SIM \\
\hline & $\begin{array}{l}\text { Escala dos profissionais com nome e horário de tra- } \\
\text { balho }\end{array}$ & Sim e disponível $=0,5 /$ Não $=0,0$ & SIM e disponível \\
\hline & Ouvidoria como canal de comunicação com o usuário & $\operatorname{Sim}=1,0 /$ Não $=0,0$ & SIM \\
\hline & $\begin{array}{l}\text { Espaço para sala de espera com cadeiras em boas } \\
\text { condições de uso e assento para pelo menos } 15 \text { pes- } \\
\text { soas }\end{array}$ & $\operatorname{Sim}=1,0 /$ Não $=0,0$ & SIM \\
\hline & $\begin{array}{l}\text { Fichas para registro do acolhimento e formulário para } \\
\text { encaminhamento a outros serviços }\end{array}$ & $\operatorname{Sim}=0,5 /$ Não $=0,0$ & SIM \\
\hline Processo & № de acolhimentos realizados & 25/mês & $\begin{array}{l}\text { No mínimo } 25 \\
\text { acolhimentos por } \\
\text { mês }\end{array}$ \\
\hline
\end{tabular}

\section{Componente: Assistência à Saúde}

\section{Subcomponente: Atendimento individual/medicação}

\begin{tabular}{|c|c|c|c|}
\hline \multirow[t]{6}{*}{ Estrutura } & Profissionais de nível superior conforme a legislação & $\operatorname{Sim}=1,5 /$ Não $=0,0$ & SIM \\
\hline & Profissionais de nível médio conforme a legislação & $\operatorname{Sim}=1,5 /$ Não $=0,0$ & SIM \\
\hline & Acesso à internet & $\operatorname{Sim}=0,5 /$ Não $=0,0$ & SIM \\
\hline & Telefone fixo funcionando & $\operatorname{Sim}=0,5 /$ Não $=0,0$ & SIM \\
\hline & $\begin{array}{l}\text { Existência de computador para os profissionais da } \\
\text { assistência }\end{array}$ & $\operatorname{Sim}=1,0 /$ Não $=0,0$ & SIM \\
\hline & Existência de impressora para a rotina do serviço & $\operatorname{Sim}=0,5 /$ Não $=0,0$ & $\operatorname{SIM}$ \\
\hline
\end{tabular}

\section{Componente: Assistência à Saúde}

Subcomponente: Atendimento individual/medicação

\begin{tabular}{|c|c|c|c|}
\hline \multirow[t]{5}{*}{ Estrutura } & Ficha que direcione a elaboração do PTS & $\operatorname{Sim}=0,5 /$ Não $=0$ & SIM \\
\hline & $\begin{array}{l}\text { Transporte acessível para realização de intervenções } \\
\text { no território }\end{array}$ & $\begin{array}{l}\text { Sim e disponível em todos os momentos }=1,5 / \\
\text { Sim, mas sempre agendado com antecedência }=1,0 / \\
\text { Não }=0,0\end{array}$ & $\begin{array}{l}\text { SIM e sempre } \\
\text { disponível }\end{array}$ \\
\hline & $\begin{array}{l}\text { Medicamentos disponíveis para dispensação aos } \\
\text { usuários de acordo com a Rename }\end{array}$ & $\begin{array}{l}100,0 \text { a } 80,0 \%=2,0 / 79,9 \text { a } 60,0 \%=1,0 / \\
59,9 \text { a } 30,0 \%=0,5 /<30,0 \%=0,0\end{array}$ & $\geq 80,0 \%$ \\
\hline & $\begin{array}{l}\text { Sistema de estoque e dispensação de medicamentos } \\
\text { no Caps }\end{array}$ & $\begin{array}{l}\text { Sim e atualizado= } 1,0 / \text { Sim, mas não atualizado }=0,5 \\
\text { Não }=0,0\end{array}$ & SIM e atualizado \\
\hline & $\begin{array}{l}\text { Local para guardar prontuários dos pacientes em boas } \\
\text { condições de uso }\end{array}$ & Sim, em boas condições de uso = 0,5/ Não $=0,0$ & $\begin{array}{l}\text { SIM e em boas } \\
\text { condições de uso }\end{array}$ \\
\hline
\end{tabular}


Quadro 2. (cont.)

\begin{tabular}{llll}
\hline Dimensão & Indicador & Pontuação & Parâmetros \\
\hline Componente: Assistência à Saúde & & \\
Subcomponente: Atendimento individual/medicação & Sim e em boas condições de uso $=0,5 /$ Não $=0,0$ & SIM e em boas \\
condições de uso
\end{tabular}

\section{Componente: Assistência à Saúde}

Subcomponente: Atenção à situação de crise

\begin{tabular}{|c|c|c|c|}
\hline \multirow[t]{2}{*}{ Estrutura } & $\begin{array}{l}\text { Existência de protocolo de atenção à situação de crise } \\
\text { no Caps }\end{array}$ & $\begin{array}{l}\text { Sim, e disponível= 1,5/ Sim, mas não disponível= } 01 \\
\text { Não = 0,0 }\end{array}$ & SIM e disponível \\
\hline & Existência de sala de repouso com no mínimo 2 camas & $\operatorname{Sim}=1,0 /$ Não $=0,0$ & SIM \\
\hline \multirow[t]{3}{*}{ Processo } & $\begin{array}{l}\text { Realização no serviço do manejo do usuário em situa- } \\
\text { ção de crise sem uso de leito de internação }\end{array}$ & $\begin{array}{l}\text { Sim, na menor parte das vezes }=2,0 / \text { Sim, na maior } \\
\text { parte das vezes }=1,5 / \text { Nenhuma crise é acolhida sem } \\
\text { uso de internação }=0,0\end{array}$ & $\begin{array}{l}\text { SIM na menor } \\
\text { parte das vezes }\end{array}$ \\
\hline & $\begin{array}{l}\text { Realização de atendimento dos usuários em situação } \\
\text { de crise em ambientes fora do serviço do Caps }\end{array}$ & $\begin{array}{l}\text { Sim, com frequência }=2,0 / \text { Sim, mas com pouca frequ- } \\
\text { ência }=1,5 / \text { Não }=0,0\end{array}$ & $\begin{array}{l}\text { SIM com frequ- } \\
\text { ência }\end{array}$ \\
\hline & $\begin{array}{l}\text { Realização de discussão de casos dos usuários em } \\
\text { situação de crise }\end{array}$ & $\begin{array}{l}\text { Sim com frequência }=2,0 / \text { Sim com menos frequên- } \\
\text { cia }=1,5 / \text { Não }=0,0\end{array}$ & $\begin{array}{l}\text { SIM com frequ- } \\
\text { ência }\end{array}$ \\
\hline
\end{tabular}

Fonte: Elaboração própria.

Caps - Centro de Atenção Psicossocial; PTS - Projeto Terapêutico Singular; Raps - Rede de Atenção Psicossocial.

Na seleção dos indicadores, considerou-se a relevância, a disponibilidade e a facilidade de obtenção, dividindo-se entre eles a pontuação de cada componente. Na definição dos critérios de julgamento, utilizaram-se os parâmetros instituídos nos instrumentos legais ou em estudos científicos e, quando inexistentes, foram criados em consonância com a rotina do serviço. O quadro 2 explicita a matriz dos 77 indicadores por componentes, 44 na dimensão 'estrutura' e 33 na dimensão 'processo', com seus respectivos critérios de julgamento.

Com o modelo lógico e a matriz finalizados, passou-se à coleta dos dados primários e secundários do município. Para obtenção dos dados primários, foram utilizadas técnicas de observação direta e entrevistas por meio de questionário semiestruturado, respondido por sete profissionais, sendo uma psicóloga, duas assistentes sociais, uma terapeuta ocupacional, uma psicopedagoga, uma psiquiatra e um coordenador do serviço. Foram incluídos na pesquisa os profissionais que estivessem trabalhando no serviço há pelo menos um ano. Além disso, realizou-se a análise de documentos, tais como livros de registros de atividades de grupos 
terapêuticos, de matriciamento, de reuniões de equipe, dentre outros. Também foram utilizados dados secundários extraídos do Sistema de Registro das Ações Ambulatoriais de Saúde. A triangulação das informações foi adotada, pois é capaz de acrescentar rigor, amplitude e profundidade à investigação ${ }^{\mathbf{1 4}}$.

Para a obtenção do GI, foram utilizados os indicadores de estrutura e processo de cada componente do modelo lógico. O cálculo do GI foi auferido pelo somatório das pontuações obtidas em relação aos valores máximos, arbitrados segundo a relevância para o objeto de estudo, por dimensão, componente e subcomponente, no caso específico da assistência à saúde. $\mathrm{O}$ GI total foi baseado no somatório de todos os indicadores. Ao final, adotou-se a seguinte classificação: implantado, quando alcançou percentuais que variaram de 80,0 a 100,0\%; parcialmente implantado, de 60,0 a $79,9 \%$; incipiente, de 40,0 a $59,9 \%$; e não implantado, abaixo de $39,9 \%$.

Para a análise dos resultados, ou efeitos, foram definidos quatro indicadores, respeitando a validade do conteúdo, relevância, disponibilidade de informação, facilidade de obtenção e simplicidade do cálculo. Elaborou-se também um indicador composto de satisfação do usuário, calculado a partir de dez questões utilizando a escala Likert de cinco pontos, que abordaram as seguintes temáticas: disponibilidade de medicamentos, qualidade do atendimento dos profissionais - psicólogo, enfermeiro, assistente social, terapeuta ocupacional - e do médico, participação nos grupos terapêuticos e instalações do Caps II (quadro 3).

Quadro 3. Indicadores de resultado do Centro de Atenção Psicossocial - Caps II. Município de Bezerros - Pernambuco, 2019

\begin{tabular}{|c|c|c|c|c|}
\hline Indicador & Método de cálculo & Parâmetro & Fonte & Média \\
\hline $\begin{array}{l}\text { *No de casos de usu- } \\
\text { ários em situação de } \\
\text { crise encaminhados a } \\
\text { outros serviços }\end{array}$ & $\begin{array}{l}\text { No de usuários em situação } \\
\text { de crise encaminhados a } \\
\text { outros serviços por mês }\end{array}$ & $\begin{array}{l}\text { Até } 1 / \text { mês, ou } 10 \% \text { dos } \\
\text { usuários cadastrados }\end{array}$ & $\begin{array}{l}\text { Relatório mensal do } \\
\text { serviço, prontuários }\end{array}$ & 0,23 \\
\hline $\begin{array}{l}\text { *\% de famílias de } \\
\text { usuários que par- } \\
\text { ticipam de grupos } \\
\text { terapêuticos }\end{array}$ & $\begin{array}{l}\text { No de núcleos familiares } \\
\text { participantes dos grupos e } \\
n^{\circ} \text { de usuários cadastrados } \\
\text { do Caps por mês }\end{array}$ & $100 \%$ & $\begin{array}{l}\text { RAAS/livro de regis- } \\
\text { tro grupos/livro de } \\
\text { registro de usuários } \\
\text { admitidos }\end{array}$ & $35,7 \%$ \\
\hline $\begin{array}{l}\text { *No de ações de ma- } \\
\text { triciamento sistemá- } \\
\text { tico realizadas pelo } \\
\text { Caps com equipes de } \\
\text { atenção básica }\end{array}$ & $\begin{array}{l}\text { № de ações de matricia- } \\
\text { mento realizadas pelos pro- } \\
\text { fissionais do Caps com as } \\
\text { equipes de atenção básica } \\
\text { do município por mês }\end{array}$ & $1 /$ mês & $\begin{array}{l}\text { RAAS, relatório } \\
\text { mensal }\end{array}$ & 1,25 \\
\hline $\begin{array}{l}\text { **Satisfação dos } \\
\text { usuários }\end{array}$ & $\begin{array}{l}\text { Média do indicador com- } \\
\text { posto }\end{array}$ & $\begin{array}{l}\geq 5=\text { muito satisfeito } \\
3,0-4,9=\text { satisfeito } \\
1,1-2,9=\text { insatisfeito } \\
\leq 1=\text { muito insatisfeito }\end{array}$ & EIC & 4,3 \\
\hline
\end{tabular}

Fonte: Elaboração própria.

Caps - Centro de Atenção Psicossocial; RAAS - Registro das Ações Ambulatoriais de Saúde.

*Período de referência: ano 2018

**Período de referência: ano 2019. Indicador construído a partir de dez questões que adotam a Likert de 4 pontos, referentes à disponibilidade de medicamentos, qualidade do atendimento do psicólogo, enfermeiro, assistente social, terapeuta ocupacional e do médico, participação dos usuários em grupos terapêuticos, condições físicas e instalações do Caps. 
Para o cálculo do indicador de satisfação do usuário, utilizou-se questionário estruturado, aplicado a uma amostra intencional de 33 usuários admitidos no serviço que apresentassem um quadro estável para responder aos questionamentos, que fossem acompanhados no Caps há pelo menos um mês e que tivessem participação regular nas atividades ofertadas no serviço. Os usuários não preencheram esses critérios foram excluídos da amostra. Realizaram-se três visitas pelos pesquisadores ao serviço no mês dezembro de 2019 para fins da pesquisa.

Por fim, imbricou-se o GI com os indicadores de resultados, confrontando-os com o modelo elaborado em um processo reflexivo dedutivo baseado na lógica do Caps com o objetivo de identificar os elementos que exerceram influência no nos resultados.

O estudo foi aprovado pelo Comitê de Ética em Pesquisa em Seres Humanos do Instituto de Medicina Integral Prof. Fernando Figueira (Imip) sob o registro 4688-15 e seguiu as recomendações da Resolução do Conselho Nacional de Saúde no 466/12 e complementares. CAAE: 09877119.4.0000.5201 - Parecer: 3.260.206. Todos os sujeitos concordaram em participar da pesquisa e assinaram o Termo de Consentimento Livre e Esclarecido.

\section{Resultados e discussão}

O Caps modalidade II do município de Bezerros foi classificado como parcialmente implantado $(74,4 \%$ ), com GI de $68,0 \%$ na dimensão estrutura e de $80,9 \%$ na dimensão processo, classificadas como parcialmente implantado e implantado, respectivamente (tabela 1). Para Alves ${ }^{15}$, um dos dispositivos estratégicos mais eficientes para a superação do modelo manicomial é o Caps. E foi com o surgimento desse dispositivo que, a partir do ano de 2015, se iniciou o processo de articulação estratégica da política de saúde mental no município de Bezerros.

Tabela 1. Grau de Implantação, por percentagem e classificação, do Centro de Atenção Psicossocial - Caps II, segundo componentes, subcomponentes e dimensões de estrutura e processo. Município de Bezerros - Pernambuco, 2019

\begin{tabular}{lcr}
\hline Componente e Subcomponente & $\%$ & Classificação \\
\hline Assistência à Saúde & $\mathbf{7 2 , 2}$ & Parcialmente implantado \\
Estrutura & 70,6 & Parcialmente implantado \\
Processo & 73,8 & Parcialmente implantado \\
\hline Acolhimento & $\mathbf{9 3 , 3}$ & Implantado \\
Estrutura & 86,6 & Implantado \\
Processo & 100,0 & Implantado \\
\hline Atendimento individual e medicação & $\mathbf{7 1 , 0}$ & Parcialmente implantado \\
Estrutura & 82,1 & Implantado \\
Processo & 60,0 & Parcialmente implantado \\
\hline Atenção à situação de crise & $\mathbf{6 3 , 1}$ & Parcialmente implantado \\
Estrutura & 50,0 & Incipiente \\
Processo & 76,2 & Parcialmente implantado \\
\hline Atendimento em grupo e oficinas terapêuticas & $\mathbf{8 1 , 4}$ & Implantado \\
Estrutura & 94,1 & Implantado \\
Processo & 68,7 & Parcialmente implantado \\
\hline
\end{tabular}


Tabela 1. (cont.)

\begin{tabular}{lrr}
\hline Componente e Subcomponente & $\%$ & Classificação \\
\hline Reabilitação Psicossocial & $\mathbf{5 2 , 0}$ & Incipiente \\
Estrutura & 40,0 & Incipiente \\
Processo & 64,0 & Parcialmente implantado \\
\hline Gestão do Caps & $\mathbf{8 4 , 4}$ & Implantado \\
Estrutura & 83,3 & Implantado \\
Processo & 85,6 & Implantado \\
\hline Mobilização social e educação na saúde & $\mathbf{6 6 , 6}$ & Parcialmente implantado \\
Estrutura & 50,0 & Incipiente \\
Processo & 83,3 & Implantado \\
\hline Total & $\mathbf{7 4 , 4}$ & Parcialmente implantado \\
Estrutura & 68,0 & Parcialmente implantado \\
Processo & 80,9 & Implantado \\
\hline
\end{tabular}

Fonte: Elaboração própria.

Caps - Centro de Atenção Psicossocial.

O subcomponente 'acolhimento' e o componente 'gestão do' foram os mais bem avaliados, tanto na estrutura como no processo, ambos considerados implantados; ao passo que 'reabilitação psicossocial' obteve a pior classificação, sendo o único avaliado como incipiente. Analisando separadamente as dimensões, os resultados mais críticos, ou seja, classificados como incipientes na estrutura, foram nos subcomponentes 'atenção à situação de crise' e 'reabilitação psicossocial', bem como o componente 'mobilização social/educação em saúde'. Já na dimensão processo, os subcomponentes 'atendimento individual/medicação', 'atenção à situação de crise', 'atendimento em grupo/oficinas terapêuticas' e 'reabilitação psicossocial' foram os piores avaliados, todos classificados como parcialmente implantado. Ainda no que diz respeito à dimensão processo, vale ressaltar que o 'acolhimento' foi o único a obter GI 100,0\%, considerado, portanto, como implantado (tabela 1).

O acolhimento propõe a inversão do modelo de atenção à saúde e objetiva organizar os serviços de forma usuário-centrado ${ }^{16}$. Recomenda-se que todo Caps possua um protocolo com as diretrizes que norteiam esse processo e que esteja disponível para todos os profissionais, que devem participar diariamente, favorecendo, assim, a construção do vínculo, da corresponsabilização e da autonomia dos usuários ${ }^{16}$. Em pesquisa realizada no Caps de Pelotas (RS), observou-se que o acolhimento produz, de fato, efeitos positivos no cotidiano do serviço, apresentando-se como um organizador dos processos de trabalho ao provocar mudanças no modelo de atenção à saúde17. Porém, como parte da função do serviço, faz-se necessário que o perfil da demanda espontânea ou referenciada do serviço seja rotineiramente analisado para que, dessa forma, o Caps possa servir de apoio à construção da linha de cuidado em saúde mental na Raps.

Quanto ao conceito de reabilitação psicossocial, há uma diversidade de concepções que se constituíram ao longo do processo de reforma psiquiátrica no Brasil. Para Pitta ${ }^{18}$, a reabilitação psicossocial é um processo que facilita ao indivíduo, a despeito de suas limitações, mais autonomia na vida social e comunitária. Ao observar esse subcomponente, a dimensão estrutura na avaliação foi considerada um ponto crítico (GI 40,0\%), podendo acarretar um não desenvolvimento pleno de iniciativas voltadas 
à reinserção social dos usuários do Caps.

Estudos mostram os desafios para implantação de rotinas e atividades inclusivas nos serviços substitutivos, que precariza a atenção psicossocial. Azevedo e colaboradores ${ }^{19}$ demonstraram que as oportunidades permanentes de atividades externas vivenciadas em um Caps estavam aquém das reais necessidades dos usuários. Organizar um serviço que opere segundo a lógica do território é encontrar e ativar os recursos locais existentes, estabelecendo alianças com grupos e movimentos de arte ou com cooperativas de trabalho para potencializar as ações de afirmação das singularidades e de participação social20.

O modelo de atenção psicossocial tem como características a valorização do saber e das opiniões dos usuários e de suas famílias na construção do PTS, e a incorporação da noção interdisciplinar ${ }^{21}$. Pensar sobre PTS em saúde mental é aproximar-se de um processo social complexo que abrange a reflexão de conceitos fundamentais para o cuidado psicossocial22. A utilização de tal dispositivo pelo serviço foi considerada uma questão crítica, uma vez que foi observado que o PTS não é realizado com todos os usuários admitidos no serviço, o que pode sugerir que sua elaboração se dá apenas para os casos de maior gravidade. Considerando esse contexto da singularidade, é fundamental que o serviço fortaleça os espaços para atendimento individual aos usuários e familiares, além da realização de visitas domiciliares que possibilitem tanto uma aproximação com a realidade vivida pelos usuários como também permitem pensar em estratégias de cuidado que a comunidade possa oferecer ${ }^{23}$.

No que se refere ao atendimento em grupos e oficinas terapêuticas, observa-se que o Caps dispõe de estrutura que favorece o desenvolvimento de tais atividades (GI 94,1\%), embora na dimensão 'processo' esse subcomponente tenha sido considerado parcialmente implantado (GI 68,7\%). O indicador "percentual de famílias de usuários que participam de grupos terapêuticos', que obteve resultado de $35,7 \%$ no ano de 2018 (quadro 3), mostrou baixa adesão das famílias nos espaços dos grupos terapêuticos. Sabe-se que as oficinas terapêuticas nos Caps possibilitam a projeção de conflitos por meio das atividades desenvolvidas, com a valorização do potencial criativo, imaginativo e expressivo do usuário e de seus familiares, além do fortalecimento da autoestima e da autoconfiança, além da expressão da subjetividade. Podem ser consideradas terapêuticas quando possibilitarem aos usuários dos serviços um lugar de fala, expressão e acolhimento ${ }^{24}$.

Nesse sentido, estudo realizado em dois Caps de Natal, Rio Grande do Norte, demonstrou que os familiares entrevistados percebiam bem o papel e a importância das oficinas terapêuticas no cenário investigado, embora tenham levantado questões a serem consideradas, como o papel das atividades desenvolvidas nos serviços, que necessitam planejamento dinâmico. As atividades devem ser articuladas com os demais domínios do conhecimento profissional segundo as necessidades e as possibilidades dos técnicos, usuários e familiares, para que seja terapêutica; favorecendo a inventividade e a produção de existências subjetivas dos usuários. A execução de atividades desconexas das necessidades do usuário transforma-se na mesma ideia de atenção focada na clínica, da doença mental, em detrimento do desenvolvimento de potencialidades do sujeito psicossocial25.

Com relação à atenção em situação da crise, este estudo mostrou que questões relacionadas à estrutura precisam ser melhoradas (GI $50,0 \%)$. O serviço não possui protocolo de atenção à crise, o que sugere que fica a cargo de cada profissional definir e desencadear a resposta mais indicada a cada caso. Embora o protocolo em saúde mental apresente limitações quanto à singularidade da atenção, facilita a comunicação entre os profissionais e orienta as práticas pelos pressupostos de evidências científicas ${ }^{\mathbf{2 6}}$. No tocante ao processo, o serviço vem desenvolvendo ações tais como o matriciamento com a atenção primária e com o serviço de urgência que podem ser consideradas um potencial no manejo da situação da crise no 
território, pautada no modelo psicossocial. De acordo com Dimenstein ${ }^{27}$, a realização do apoio matricial é capaz de, em alguns casos, evitar situações de crise ou abordá-las antes que se tornem mais graves, bem como evitar internações psiquiátricas arbitrárias.

No campo da gestão do Caps, tanto a estrutura como o processo foram considerados implantados (GI 83,3\% e 85,6\%, respectivamente). O serviço dispõe de protocolos e diretrizes que norteiam o processo de trabalho, além de realizar planejamento, monitoramento e discussão de casos. $\mathrm{O}$ único indicador da dimensão 'processo' que não foi satisfatório refere-se à realização de supervisão clínica no serviço, que, vale salientar, é um dispositivo muito importante na mudança da gestão do trabalho em saúde mental, uma vez que discute questões clínicas e institucionais dos serviços e da rede de atenção psicossocial ${ }^{28}$.

Cartografia realizada em uma área programática do município do Rio de Janeiro mostrou os efeitos transversais da supervisão clínico-institucional na Raps. O referido dispositivo do cuidado contribuiu para ativar o sistema de regulação, ou seja, a rede de serviços em sua dinâmica acêntrica, funcional, afetiva, intensiva, pública e criativa, bem como ativou a rede de usuários, trabalhadores e gestores que constroem coletivamente o cuidado e a gestão do cuidado no território ${ }^{29}$.

A mobilização social e educação na saúde foram avaliadas na dimensão 'estrutura' como incipiente (GI 50,0\%) e na dimensão 'processo' como implantada (GI 83,3\%), o que revela que, mesmo com estrutura incipiente, o serviço consegue avançar nas atividades do movimento popular pela saúde mental, bem como no fortalecimento de espaços coletivos para analisar e refletir sobre as práticas profissionais, ambos de fundamental importância para a transição paradigmática do modelo asilar para o modo psicossocial ${ }^{30}$.

Estudos mostraram ter sido possível reafirmar o potencial da educação permanente como constitutiva e qualificadora do processo de trabalho em saúde, contribuindo, em especial, com os Caps ao indicar características que valorizem suas atividades. Um exemplo são as tecnologias de trabalho eficazes na qualificação da atenção à saúde prestada e que podem ser desenvolvidas no cotidiano dos diversos serviços de saúde mental, como um modo de resistência ao enfrentamento dos atuais desafios do Sistema Único de Saúde ${ }^{31}$.

Com relação aos indicadores de resultado, três deles estavam dentro do parâmetro de referência estabelecido e demonstraram coerência entre o GI e os efeitos, especialmente no que diz respeito ao processo. $\mathrm{O}$ fato de a estrutura do Caps II de Bezerros ainda apresentar algumas deficiências, elas não exerceram influência direta nos resultados. O indicador 'número de casos de usuários em situação de crise encaminhados a outros serviços' alcançou média 0,23; o 'número de ações de matriciamento sistemático realizadas pelo com equipes de atenção básica' alcançou média 1,25; e o indicador de 'satisfação dos usuários’ atingiu média 4,3. A exceção se deu ao 'percentual de famílias de usuários que participam de grupos terapêuticos', com 35,7\% indicando baixa adesão das famílias (quadro 3).

A satisfação dos usuários é um indicador útil de ser avaliado porque ele também pode influenciar aspectos importantes no tratamento. Além disso, serve para indicar até que ponto os tratamentos oferecidos e as condições dos serviços são socialmente aceitáveis ${ }^{32}$. Assim, a satisfação dos usuários do Caps II de Bezerros foi positiva e considerou aspectos referentes à disponibilidade de medicamentos, qualidade do atendimento do psicólogo, enfermeiro, assistente social, terapeuta ocupacional e do médico, da participação dos usuários em grupos terapêuticos e das condições físicas e instalações.

Outros estudos já indicavam que a satisfação dos usuários de serviços de saúde mental está relacionada às questões de acesso, como a disponibilidade de serviços gratuitos, continuidade dos cuidados e isenção de custos com medicamentos. Entretanto, para que a proposta dos Caps ultrapasse esses critérios, além da garantia do acesso, é preciso que os 
usuários se sintam acolhidos e satisfeitos, a fim de que possam construir junto com a equipe $o$ PTS, respeitando sua subjetividade e a possibilidade de estabelecer vínculo com o serviço ${ }^{33}$.

Algumas limitações foram observadas neste estudo, especialmente no que concerne à seleção de indicadores de resultado. A operacionalização de indicadores em saúde mental é tarefa que impõe desafios, uma vez que a tradição de indicadores nessa área é mais restrita quando comparada a outros campos da saúde ${ }^{34}$. A indisponibilidade de indicadores e a ausência de um sistema de informação no serviço para a obtenção de informações confiáveis foram as maiores limitações deste estudo, sendo necessário recorrer a registros em livros e relatórios do serviço.

\section{Conclusões}

Os resultados obtidos neste estudo indicaram que a estrutura do serviço do Caps foi considerada parcialmente implantada, principalmente no que diz respeito à atenção em situação de crise, reabilitação psicossocial e mobilização social e educação na saúde, que são questões importantes para a consolidação do modelo de atenção psicossocial. A baixa disponibilidade de transportes para os profissionais do serviço dificulta a continuidade da realização de ações no território, bem como a realização de atividades extramuros com os usuários.

As diretrizes da Raps reforçam a necessidade da autonomia dos usuários para o exercício da cidadania, e o ambiente onde eles podem exercer essa função é justamente a comunidade, em contato com instituições, grupos organizados e pessoas com as quais convivem ${ }^{35}$. $\mathrm{O}$ baixo investimento em formação continuada dos profissionais, bem como a baixa adesão e participação em eventos de discussão da saúde mental também dificultam a consolidação do modelo de atenção.

Apesar de reconhecidos os avanços da política de saúde mental no Brasil, seja no aumento da oferta dos serviços substitutivos, na transferência de gastos realizados no atendimento comunitário, na melhoria da qualidade de vida de usuários e familiares ou na desconstrução gradativa do estereótipo da loucura ${ }^{36}$, este estudo demonstra que ainda persistem problemas estruturais e no processo de trabalho.

Avançando gradativamente em cada região, a implantação e o funcionamento do Caps perpassam muitos desafios. No entanto, é fato que somente construir uma infraestrutura diferente do manicômio, com todo um arsenal de trabalho em grupo, oficinas terapêuticas diversas, e operar mudanças legais no cenário da saúde mental não são o bastante. É nos processos de cogestão, atuação em rede, qualificação contínua e suporte mútuo que os gestores encontram possibilidades de lidar com as adversidades e construir soluções, uma vez que o campo da saúde mental possui peculiaridade no seu fazer que o torna distinto de outras práticas de saúde ${ }^{37}$.

Faz-se necessário investir nos profissionais de saúde e em uma nova forma de cuidado e assistência comunitária, pois esses profissionais atuam no transcurso da Reforma Psiquiátrica, mas desconhecem sua existência e não contemplam a discussão em sua formação, cujo fazer cotidiano coloca a necessidade de um trabalho interdisciplinar, de formas plurais de intervir e cuidar do sujeito com transtorno mental.

Diante das dificuldades encontradas, sugere-se que o serviço amplie o desenvolvimento de iniciativas articuladas com os recursos do território e que visem a promover o protagonismo para o exercício dos direitos de cidadania de usuários e familiares e que, para superar os desafios estruturais, captem recurso financeiro de custeio do governo federal.

Contudo, ressalta-se que a utilização de informações oriundas apenas da avaliação feita pelos profissionais poderia conduzir, segundo Perreault e colaboradores ${ }^{38}$, a uma deterioração da qualidade e da pertinência das informações. Assim, optou-se pela inclusão do indicador de satisfação do usuário, e, a partir de duas perspectivas diferentes e complementares, se 
chegar a uma avaliação mais completa e mais ampla dos efeitos e da qualidade do serviço.

Avaliar sistemática e amplamente os resultados advindos desse processo de reforma assistencial em saúde mental torna-se importante no sentido de reforçar os acertos e corrigir as deficiências, bem como de subsidiar novos processos políticos decisórios. Fazer isso por meio de pesquisas avaliativas em saúde, que sigam minimamente os padrões esperados de utilidade - fornecimento de dados relevantes e necessários - e acurácia - serem capazes de informar validamente sobre o mérito das práticas e processos avaliados -, além de terem exequibilidade e condução ética, parece ser o melhor caminho $^{39}$. Espera-se, portanto, que este estudo, somado aos demais já publicados, contribua para o reconhecimento da importância dos processos avaliativos dos Caps.

\section{Colaboradores}

Silva GM (0000-0002-4086-1767)* realizou a coleta e análise dos dados, participou da concepção e planejamento do estudo, redação do manuscrito e aprovação da versão final do artigo. Felisberto E (0000-00022316-2251)* participou da concepção e planejamento do estudo e colaborou na redação, revisão crítica e aprovação da versão final do artigo. Samico I (0000-0002-8338-7946)* participou da concepção e planejamento do estudo e colaborou na redação, revisão crítica e aprovação da versão final do artigo. Albuquerque AC (0000-0001-6305-4127)* participou da análise dos dados, concepção e planejamento do estudo, revisão crítica e aprovação da versão final do artigo. 


\section{Referências}

1. World Health Organization. World health statistics 2018: monitoring health for the SDGs, sustainable development goals. Genebra: WHO; 2018.

2. Brasil. Ministério da Saúde, Secretaria de Atenção à Saúde Mental, Departamento de Ações Programáticas Estratégicas. Política Nacional de Saúde Mental. Brasília, DF: MS, 2008.

3. Saraceno B. Libertando identidades: da reabilitação psicossocial à cidadania possível. 2. ed. Rio de Janeiro; 2001

4. Pitta A. Os Centros de Atenção Psicossocial: espaços de reabilitação? J bras Psiquiatria. 1994; 43(12):647655.

5. Brasil. Lei $n^{0} 10.216$, de 6 de abril de 2001. Dispõe sobre a proteção e os direitos das pessoas portadoras de transtornos mentais e redireciona o modelo assistencial em saúde mental. Diário Oficial da União. 6 Abr 2001. [acesso em 2021 jan 21]. Disponível em: http://www.planalto.gov.br/ccivil_03/leis/leis_2001/ 110216.htm.

6. Brasil. Ministério da Saúde, Secretaria de Atenção à Saúde, Departamento de Ações Programáticas Estratégicas. Brasília, DF: MS, 2015. (Cadernos HumanizaSUS - V. 5, Saúde Mental).

7. Brasil. Ministério da Saúde, Secretaria de Atenção à Saúde, Departamento de Ações Programáticas Estratégicas. Saúde mental no SUS: os centros de atenção psicossocial. Brasília, DF: MS; 2004.

8. Sampaio JJC, Guimarães JMX, Carneiro C, et al. O trabalho em serviços de saúde mental no contexto da reforma psiquiátrica: um desafio técnico, político e ético. Ciênc. Saúde Colet. 2011; 16(12): 4685-4694.

9. Bessa M, Azevedo DC, Filho JD. Assistência de saúde mental em um Centro de Atenção Psicossocial: avaliação na perspectiva profissional. JONAH. 2016; 6(2):279-86.
10. Champagne F, Brousselle A, Hartz Z, et al. A Análise da Implantação. In: Brousselle A, Brousselle A, Hartz $Z$, et al., editores. Avaliação em saúde: Conceitos e Métodos. Rio de Janeiro: Fiocruz; 2011. p. 217-238.

11. Yin RK. Estudo de caso: planejamento e métodos. 5. ed. Porto Alegre: Bookman; 2015.

12. Brasil. Ministério da Saúde. Portaria $n^{\circ} 336$, de 19 de fevereiro de 2002. Institui as modalidades dos Centros de Atenção Psicossocial, no âmbito do Sistema único de Saúde. Diário Oficial da União [internet]. 19 Fev 2002. [acesso em 2021 jan 22]. Disponível em: https://bvsms.saude.gov.br/bvs/saudelegis/gm/2002/ prt0336_19_02_2002.html.

13. Donabedian A. The definition of quality: a conceptual exploration. In: Arbor A, organizador. The definition of quality and approaches to its assessment. Michigan: Health Administration Press; 1980. p. 3-31.

14. Denzin NK, Lincoln Y. The discipline and practice of qualitative research. In: Denzin NK, Lincoln Y, orgnaizadores. Handbook of qualitative research. Thousand Oaks: Sage Publications; 2000. p. 1-28.

15. Alves DS. Integralidade nas políticas de saúde mental. In: Pinheiro R, Mattos RA. Os sentidos da integralidade na atenção e no cuidado à saúde. Rio de Janeiro: IMS, Abrasco; 2001. p. 167-176.

16. Brognoli FF, Rodrigues J. Acolhimento no serviço de atenção psicossocial. Cad bras Saúde Mental. 2014; 6(13):61-74.

17. Scheibelb A, Ferreira LH. Acolhimento no CAPS: reflexões acerca da assistência em saúde mental. RBSP. 2011; 4(35):966-983

18. Pitta A. O que é a reabilitação psicossocial no Brasil, hoje? In: Pitta A, organizador. Reabilitação psicossocial no Brasil. São Paulo: Hucitec; 1996. p. 19-26.

19. Azevedo DM, Silva GWS, Miranda FAN, et al. Percepções de profissionais de saúde sobre inclusão social 
em um Centro de Atenção Psicossocial. Rev Rene. 2019; 8(20):335-337.

20. Amorim AKMA, Severo AKS. Saúde mental, cultura e arte: discutindo a reinserção social de usuários da Rede de Atenção Psicossocial. Rev. Interinstitucional de Psicologia. 2019; 12(2):282-299.

21. Campos GWS, Amaral MA. A clínica ampliada e compartilhada, a gestão democrática e redes de atenção como referenciais teórico-operacionais para a reforma do hospital. Ciênc. Saúde Colet. 2007; 12(4):84959.

22. Oliveira GN. O Projeto Terapêutico e a mudança nos modos de produzir saúde. São Paulo: Hucitec; 2010.

23. Reisdorfer E, Cardoso L, Pereira SS, et al. Visita domiciliar aos pacientes portadores de transtorno mental: ampliando as opções terapêuticas possíveis em um serviço ambulatorial. Sau. \& Transf. Soc. [internet]. 2014 [acesso em 2020 fev 4]; 91-95. Disponível em: http://pepsic.bvsalud.org/scielo.php?script=sci arttext\&pid=S2178-70852014000100014.

24. Thofehrn MB, Andrade APM, Carvalho LA, et al. Oficina terapêutica como expressão da subjetividade. SMAD [internet]. 2016 [acesso em 2020 fev 5]; 147-153. Disponível em: http://pepsic.bvsalud.org/scielo.php?script=sci_arttext\&pid=S1806$9762016000300003 \& \operatorname{lng}=$ pt\&nrm=iso\&tlng=pt.

25. Azevedo DM, Miranda FAN. Oficinas terapêuticas como instrumento de reabilitação psicossocial: percepção de familiares. Esc. Anna Nery. 2011; 15(2): 339345 .

26. Werneck MAF, Faria HP, Campos KFC. Protocolos de cuidado à saúde e de organização do serviço. Belo Horizonte: UFMG; 2009.

27. Lima M, Dimenstein M. O apoio matricial em saúde mental: uma ferramenta apoiadora da atenção à crise. Interface. Butucatu. 2016; 20(58):625-35.

28. Onocko Campos RT, Severo AKS, L'Abbate S. A supervisão clínico-institucional como dispositivo de mu- danças na gestão do trabalho em saúde mental. Interface Comum Saúde Educ [internet]. 2014 [acesso 2020 jan 18]; 545-556. Disponível em: http://www. scielo.br/scielo.php?script=sci_abstract\&pid=S141432832014000300545\&lng=en\&nrm=iso\&tlng=pt.

29. Oliveira JAM, Passos E. Efeitos transversais da supervisão clínico-institucional na Rede de Atenção Psicossocial. Polis e Psique. [internet]. 2012 [acesso em 2020 jan 18]; 171-187. Disponível em: https://seer. ufrgs.br/PolisePsique/article/view/40326.

30. Pegoraro RB, Rabelo IVM, Zanini DC, et al. Concepções sobre o modo de atenção psicossocial de profissionais da saúde mental de um CAPS. Rev. Psicol. Saúde. 2015; 7(2):161-167.

31. Pinheiro MCC, Hypólito ALM, Kantorski LP. Educação permanente no processo de trabalho em saúde mental. J. nurs. health. 2019; 9(2):199-203.

32. Ruas CM, Lima MG, Silva SN. Avaliação de Serviços de Saúde Mental Brasileiros: satisfação dos usuários e fatores associados. Ciênc. Saúde Colet. 2018; 23(11): 3799-3810.

33. Dutra VFD, Oliveira RMP. Revisão integrativa: as práticas territoriais de cuidado em saúde mental. Aquichan. 2015; 15(4):529-540.

34. Campos RTO, Furtado JP. Entre a saúde coletiva e a saúde mental: um instrumental metodológico para avaliação da rede de Centros de Atenção Psicossocial (CAPS) do Sistema Único de Saúde. Ciênc. Saúde Colet. 2006; 22(5):1053-1062.

35. Bielemann VLM, Kantorski LP, Borges LR, et al. A inserção da família nos centros de atenção psicossocial sob a ótica de seus atores sociais. Texto contexto enferm. 2009; 18(1):131-39.

36. Campos Onocko RT, Furtado JP, Trapé TL, et al. Indicadores para avaliação dos Centros de Atenção Psicossocial tipo III: resultados de um desenho participativo. Saúde debate. 2017; 41(11):71-83.

37. Pitta AMF, Lancman S, Kinoshita RT, et al. Determi- 
nantes da qualidade de serviços de saúde mental em municípios brasileiros - Estudo da satisfação com os resultados das atividades desenvolvidas por pacientes, familiares e trabalhadores dos serviços. $\mathrm{J}$ bras Psiquiatr. 1996; 44(9):441-452.

38. Perreault M, Katerelos TE, Sabourin E, et al. Information as a distinct dimension for satisfaction assessment of outpatient psychiatric services. Int J Health Care Qual Assur Inc Leadersh Health Serv. 2001; 14(3):111-20.
39. Hartz ZMA. Avaliação dos programas de saúde: perspectivas teórico-metodológicas e políticas institucionais. Ciênc. Saúde Colet. 1999; 4(2):341-353.

Recebido em 27/02/2020

Aprovado em 03/07/2020

Conflito de interesses: inexistente

Suporte financeiro: não houve 